\title{
The effect of Au and Pt nanoclusters on the structural and hydrogen sensing properties of $\mathrm{SnO}_{2}$ thin films
}

\author{
I. Fasaki ${ }^{1}$, M. Suchea $2, a$, T.Platanias $^{1}$, G. Mousdis ${ }^{1, *}$, G. Kiriakidis ${ }^{2, b}$, M. Kompitsas $^{1}$ \\ ${ }^{1}$ National Hellenic Research Foundation, Theoretical and Physical Chemistry Institute, Vasileos Konstantinou Ave. 48, 11635 \\ Athens, Greece \\ ${ }^{2}$ IESL-FORTH and University of Crete a) Chemistry Dept. and Physics Dept. , P.O. Box 1385, Voutes, 71110 Heraklion, Greece \\ * e-mail: gmousdis@eie.gr tel.: +302107273838
}

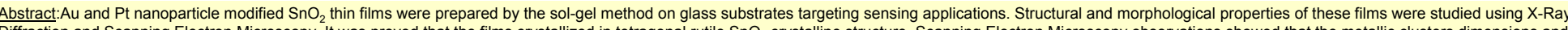

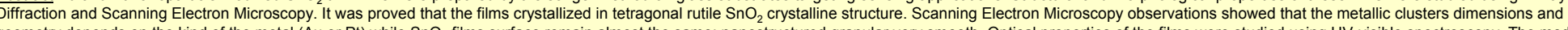

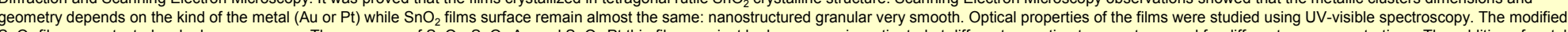

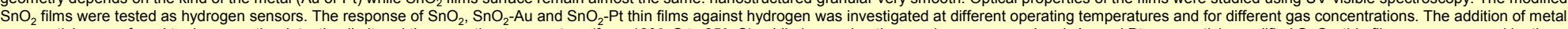

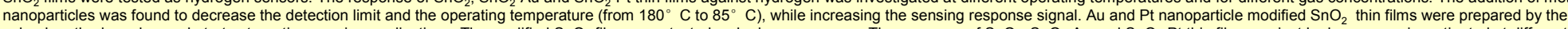

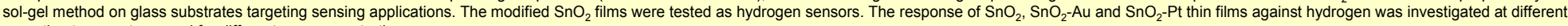

\section{$\underline{\text { Structural, morphological and optical properties of } \mathrm{SnO}_{2}} \underline{\underline{\text { thin films }}}$}

$X R D$ characterization tendency of texturing in the [101] and [200] crystalline directions of the tetragonal rutile structure.

- $\mathrm{SnO}_{2}-\mathrm{Pt}$ film has shown a weaker tendency for texturing with a preferred
towards the [200].

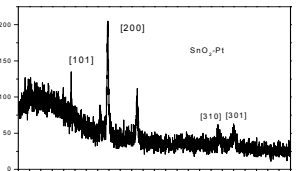
- This is a clear indication of the catalytic effect that these growth structure of the $\mathrm{SnO}_{2}$
SEM characterization
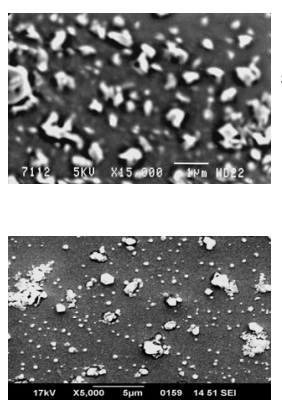

Transmittance and Reflection spectra

\section{Hydrogen sensing properties}

Results and Discussion

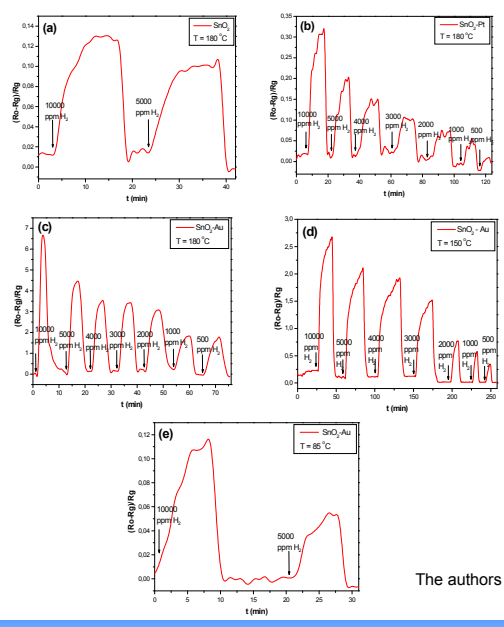

\section{Sensing mechanism}

-Hydrogen sensing tests were performed in an aluminum vacuum test chamber. The hydrogen concentration was calculated on the basis on the partial pressures of the sensing gas and dry air inside the chamber. A bias of $1 \mathrm{~V}$ was applied, and the current through the hydrogen sensing in real time.

- The sensitivity was defined as: $\mathrm{S}=(\mathrm{Ro}-\mathrm{Rg}) / \mathrm{Rg}$ where $\mathrm{Ro}$ is the resistance of the film in dry air and $\mathrm{Rg}$ is the resistance of the film in the gas/air mixture

-It is clear that the sensitivity of all $\mathrm{SnO} 2$ sensors towards hydrogen showed a strong dependence on both the operating temperature and gas concentration. For the same operating temperature, the response of gas concentration.

- Modifying $\mathrm{SnO}_{2}$ with Au nanoparticles decreased the sensor workin temperature with respect to the unmodified one from 180 down to $85^{\circ} \mathrm{C}$, while the response increased almost by 50 times at the temperature
then possible.

-At $180{ }^{\circ} \mathrm{C}$ the $\mathrm{SnO}_{2}$ films have shown a response of about an order of magnitude while this was enhanced distinctly by the presence of $\mathrm{Pt}$ in the $\mathrm{SnO}_{2}$-Pt modified films.
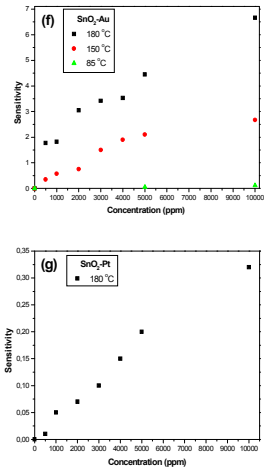

-The atmospheric oxygen adsorption may remove electrons from the conduction band, resulting in the formation of localized either $\mathrm{O}^{-}$or $\mathrm{O}_{2}-$ depending on the the hydrogen gas splits up into hydra ( $\mathrm{Pl}$ or $\mathrm{Au}$ ) which form protons by donating the electrons to the conduction band of nanocrystalline doped $\mathrm{SnO}_{2}$ film.

-The electron gain increases the film conductivity, which is reflected in the drop in the initial sensor resistance. The generated protons get associated with the surface adsorbed oxygen-ions ( $\mathrm{O}^{-}$or $\mathrm{O}_{2}{ }^{-}$ions) and hop from one oxygen-ion to another. During this process, the two adjacent $\mathrm{OH}$-groups may condense to form $\mathrm{H}_{2} \mathrm{O}$ that evaporates. The overall reaction can be summarized as:

$$
\mathrm{H}_{2}+\mathrm{O}^{-} \rightarrow \mathrm{H}_{2} \mathrm{O}+\mathrm{e}^{-}
$$

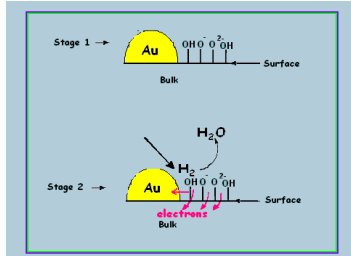

